\title{
COUCHES CONDUCTRICES ET TRANSPARENTES OBTENUES PAR PULVERISATION D'UNE CIBLE DE Sn $\mathrm{O}_{2}$
}

\author{
J. LAUNEY et A. TOSSER \\ Laboratoire d'Electronique et d'Automatique, U.E.R. des Sciences et Techniques, \\ 4006-76 007-Le Havre Cédex, France
}

(Received May 12, 1975)

\begin{abstract}
$\mathrm{SnO}_{2}$ is reactively r.f. sputtered to obtain conductive thin films of good optical transmission. Data about thermal ageing in vacuum, thermal evolution of conductivity, spontaneous ageing in air and optical transmission show how it is possible to obtain a transmission of $80 \%$ in visible light with resistance square below $1 \mathrm{k} \Omega / \mathrm{sq}$; the possibility of a multiple-step process of sputtering is presented. A "nucleation-growth" model allows an explanation of the observed phenomena.
\end{abstract}

\begin{abstract}
La pulvérisation haute-fréquence d'une cible de $\mathrm{SnO}_{2}$ en présence d'argon et d'oxygène permet d'obtenir des couches minces conductrices de bonne transparence optique. A partir des phénomènes de recuit sous vide, d'évolution de la résistance avec la température, du vieillissement spontané à l'air et de la variation de la transparence optique, il est possible de déterminer la méthode permettant d'obtenir un facteur de transmission de $80 \%$ en lumière visible et une résistance carrée inférieure à $1 \mathrm{k} \Omega / \mathrm{sq}$; l'intérêt d'une pulvérisation fractionnée est explicité. Le modèle de "germination-croissance" permet une description convenable des phénomènes observés.
\end{abstract}

\section{INTRODUCTION}

En vue de la réalisation d'éléments conducteurs non opaques de grande surface, des progrès technologiques récents ont permis de substituer des verres conducteurs aux verres isolants recouverts d'une couche conductrice transparente; mais dans le domaine de la microélectronique, les couches minces conductrices et optiquement transparentes sont encore appelées à intervenir dans la réalisation de couches minces dont l'absorption ne soit pas trop importante en lumière visible ou ultraviolette; cette dernière caractéristique est essentielle dans les dispositifs de conversion d'énergie solaire.

Aussi, divers auteurs ont procédé à des expérimentations concernant des couches d'oxyde de cadmium $^{1-3}$ et, d'indium ${ }^{2-4}$ parfois associés à de l'oxyde d'étain $3,5-12$

Les couches minces d'oxyde d'étain peuvent être préparées par hydrolyse du chlorure stannique par la vapeur d'eau13-16, par évaporation de $\mathrm{SnO}_{2}{ }^{17-19}$ ou par pulvérisation non réactive de $\mathrm{SnO}_{2}{ }^{4}$, la méthode la plus répandue étant la pulvérisation d'une cathode d'étain sous haute tension continue, dans un mélange Oxygène-Argon où la proportion d'oxygène varie de 5 à $50 \%$. Cette dernière méthode est préférée en raison de la facilité qu'elle procure pour la préparation de structures multicouches sans rupture d'atmosphère contrôlée même si la conductibilité est inférieure à celle des dépots obtenus par voie chimique; de plus, la pulvérisation réactive fournit généralement des dépots ayant une meilleure transparence optique; les auteurs qui utilisent un faible taux d'oxygène estiment judicieux de procéder à une oxydation préliminaire de la cathode d'etain d'etain ${ }^{21,27}$; on a ainsi été conduit à utiliser des cathodes d'oxyde d'étain, qui peuvent être pulvérisées en atmosphère réactive aussi bien sous haute tension continue $^{30}$ que sous haute tension haute fréquence 29 .

La résistance électrique des dépôts obtenus apres la pulvérisation est souvent très élevée: de $500 \mathrm{k} \Omega$ pour $15 \mathrm{~min}$ de durée de pulvérisation $21 \mathrm{k} \Omega$ à $30 \mathrm{k} \Omega$ pour $3 \mathrm{~min}^{29}$; la plupart des auteurs completent donc la préparation des dépôts par un traitement thermique à l'air libre, de durée variable, et à une température élevée afin d'améliorer à la fois la conductivité et la transparence ce qui peut présenter cependant un risque de détérioration de la structure sous-jacente.

Pour obtenir une meilleure conductance, on peut être tenté de préparer des films épais, mais comme 
certains auteurs ${ }^{22}$, nous avons rencontré des difficultés en ce qui concerne en particulier l'adhérence des couches, comme l'indique Holland 31.

La complexité du procédé nous a conduit à écarter la pulvérisation d'une cathode dopée $23,27,29$ qui permet de retrouver d'aussi bons résultats que la méthode chimique $(200 \Omega$ pour $3500 \AA, 10 \mathrm{k} \Omega$ pour $50 \AA)$.

Etant donné que les procédés de pulvérisation haute fréquence (RF) assurent une très bonne reproductibilité des caractéristiques électriques de couches minces métalliques ou diélectriques à condition de faire suivre la pulvérisation d'une stabilisation thermique convenable ${ }^{32,33}$, nous avons choisi de préparer des couches non dopées de $\mathrm{SnO}_{2}$ par pulvérisation cathodique RF en milieu gazeux réactif à forte teneur en oxygène.

Nous avons tenté de définir les conditions de pulvérisation et de recuit qui conduisent à une bonne transparence optique et à une faible résistivité.

\section{TECHNIQUES EXPERIMENTALES}

Le substrat est une lame de Vycor polie optiquement de dimensions $100 \mathrm{~mm} \times 18 \mathrm{~mm} \times 3 \mathrm{~mm}$; la face apparente du substrat est en partie masquée par un pochoir en cuivre photogravé délimitant la forme de l'échantillon que l'on souhaite obtenir. Des électrodes de contact en Nickel, destinées à mesurer la résistance et à permettre le passage de faibles courants, sont déposées par pulvérisation cathodique; nous les avons préférées aux électrodes d'Or évaporées thermiquement qui même après un recuit sous vide à $250^{\circ} \mathrm{C}$ sont altérées au cours de la pulvérisation de l'oxyde d'étain; les électrodes de Nickel sont recuites pendant $4 \mathrm{~h}$ à $380^{\circ} \mathrm{C}$, température nettement supérieure à la température minimale de recuit qui est de $250^{\circ} \mathrm{C}^{11,27,34}$.

Le substrat est encastré dans la rainure d'un bloc de cuivre qui peut être chauffé électriquement ou refroidi par une circulation d'air ou d'eau; les fils de mesure sont fixés avant toute pulvérisation. Ce montage étant effectué, le substrat n'est plus manipulé jusqu'à la fin de l'étude même quand on procède à des pulvérisations successives et à des recuits successifs ou multiples avec des retours intermédiaires à la pression atmosphérique.

Le support du substrat est fixé sur une platine dotée de passages électriques et mécaniques qui clôt une enceinte cylindrique de verre où le vide est créé par une pompe à diffusion d'huile comportant un baffle optiquement opaque refroidi à l'eau; le vide limite obtenu est de quelques $10^{-6}$ Torr.

La cible qui subit la pulvérisation est une cathode en oxyde d'étain, de $99,8 \%$ de pureté, de $100 \mathrm{~mm}$ de diamètre et $6 \mathrm{~mm}$ d'épaisseur; le champ électrique radiofréquence permettant la pulvérisation est à la fréquence de 13,6 MHz et l'amplitude de la tension alternative est de $1,9 \mathrm{kV}$.

La distance entre la cathode et le substrat est de $52 \mathrm{~mm}$. Un écran métallique mobile autour d'un axe vertical permet de commencer le dépôt d'oxyde par pulvérisation seulement lorsque les parametres déterminant le processus sont stables; le délai ainsi introduit est de quelques minutes et il ne correspond pas à une prépulvérisation de $30 \mathrm{~min}$ comme celles pratiquées par certains auteurs $21,27,29$.

Lorsque le milieu gazeux est de l'argon pur, les couches de $\mathrm{SnO}_{2}$ obtenues présentent à la lumière du jour une couleur jaune ou brune, ce qui avait déjà été observé $20,21,25$; lorsque le mélange gazeux dans lequel se produit la décharge comporte $70 \%$ d'Argon et $30 \%$ d'Oxygène (la pression totale étant voisine de $2,5 \times 10^{-3}$ Torr), elles possedent de facon reproductible une résistivité plus faible et une meilleure transparence optique.

A l'aide d'une vanne de réglage, nous obtenons une pression totale réglable entre $7 \times 10^{-4}$ et $9 \times 10^{-3}$ Torr de stabilité suffisante pendant des durées supérieures à 30 minutes.

La pression utilisée pendant la pulvérisation est maintenue, lorsque le dépôt est effectué, pendant le temps de refroidissement du substrat, dont l'élévation de température est limitée à $40^{\circ} \mathrm{C}$ par une circulation d'eau.

Lors des essais thermiques, la température de la couche est repérée par un thermocouple en contact thermique avec le bloc de cuivre dans lequel est encastré le substrat; la longue durée des essais permet, malgré la résistance thermique du substrat, d'effecteur une mesure de la température de la couche à $5 \%$ près.

Le taux de pulvérisation est voisin de $92 \AA / \mathrm{min}$; cette valeur a été déterminée à partir de mesures interférométriques d'épaisseur; dès que la durée de pulvérisation dépasse $2 \mathrm{~min}$, ce taux est invariant.

\section{RESULTATS EXPERIMENTAUX}

\subsection{La pulverisation fractionée}

Les couches de $\mathrm{SnO}_{2}$ que nous obtenons sont d'une transparence optique satisfaisante; elles présentent à la lumière du jour une teinte legèrement grise de facon 


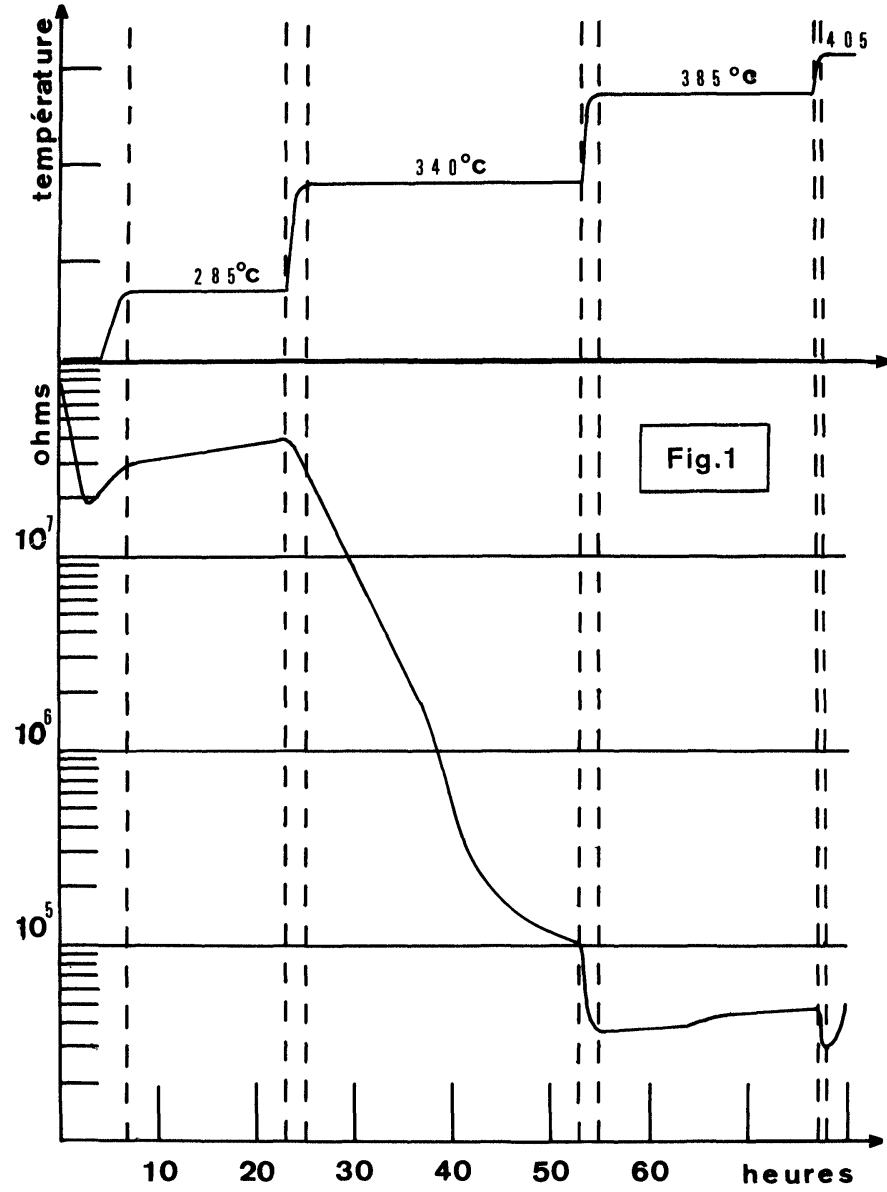

FIGURE 1 Variation de la température et de la résistance d'une couche en fonction du temps.

régulière; en ce qui concerne la résistivité elle est assez élevée et varie fortement avec la durée de la pulvérisation.

Nous avons ainsi été conduits à préparer des multicouches résultant de plusieurs opérations successives de pulvérisation. Ayant observé le rôle bénéfique d'un recuit pour l'amélioration de la conductivité, et afin de séparer les phénomènes mécaniques des phénomènes physico-chimiques superficiels intervenant lors de la stabilisation de la conductivité, nous avons étudié séparément le recuit dans une atmosphère déterminée et les effets ultérieurs de vieillissement spontané à l'air.

\subsection{Les conditions de recuit sous vide}

Nous avons délibérément écarté le traitement thermique en présence d'air qui nous semble difficile à contrôler et de reproductibilité mal définie, notamment en raison des variations du degré hygrométrique; aussi, tous les cycles thermiques de recuit sont effectués dans l'enceinte à vide, sous une pression qui demeure toujours inférieure à $3 \times 10^{-5}$ Torr.

Nous observons d'abord que, quelle que soit la résistivité initiale des couches obtenues, la résistivité reste elevée, et peut même augmenter, si la température de recuit est inférieure à $245^{\circ} \mathrm{C}$, même si le traitement dure plusieurs heures; de plus la température de recuit ne peut dépasser $400^{\circ} \mathrm{C}$ car il se produit alors sous vide une évaporation partielle de la couche.

Entre ces deux limites de $245^{\circ} \mathrm{C}$ et $400^{\circ} \mathrm{C}$, nous avons déterminé la température optimale de recuit en procédant à une montée en température par paliers successifs, de durée suffisante pour permettre la stabilisation de la résistance. La Figure 1 illustre ce processus; de facon générale, la résistance de la couche diminue très rapidement pour une température de recuit dépassant $315^{\circ} \mathrm{C}$ à $340^{\circ} \mathrm{C}$, suivant les épaisseurs de couches; la température maximale de recuit a donc été fixée à $380^{\circ} \mathrm{C}$ et nous avons réalisé un dispositif électrique de programmation automatique du cycle thermique afin que toutes les couches subissent ainsi le même traitement typique de recuit sous vide (Figure 2).

Cette valeur de la température de recuit est voisine de la valeur de $400^{\circ} \mathrm{C}$ adoptée par l'auteur qui procède à un traitement sous vide. ${ }^{29}$

L'enchaînement des opérations de préparation est tel qu'une couche n'est remise à l'air ambiant qu'après avoir subi ce recuit et qu'aucun substrat vierge, ou déjà recouvert, ne recoit un dépôt sans avoir été dégazé sous vide.

\subsection{Les variations de la resistance avec la temperature}

Chaque couche ayant été stabilisée sous vide à la température maximale de recuit pendant plusieurs heures, nous relevons ensuite in situ les courbes de variation de la résistance $\mathrm{R}$ en fonction de la température $\mathrm{T}$ entre $380^{\circ} \mathrm{C}$ et $20^{\circ} \mathrm{C}$.

Nous avons reproduit sur la Figure 3 les courbes de variations de la résistance $R$, en échelle logarithmique, en fonction de $10^{3} \times \mathrm{T}^{-1}$ où $\mathrm{T}$ est exprimé en Kelvin.

Dans la partie linéaire, qui correspond aux températures $\mathrm{T}$ inférieures à $500 \mathrm{~K}$, les pentes obtenues correspondent à une énergie d'activation s'écartant de moins de $10 \%$ de la valeur $0,035 \mathrm{eV}$ pour toutes les couches obtenues par plusieurs pulvérisations successives. 


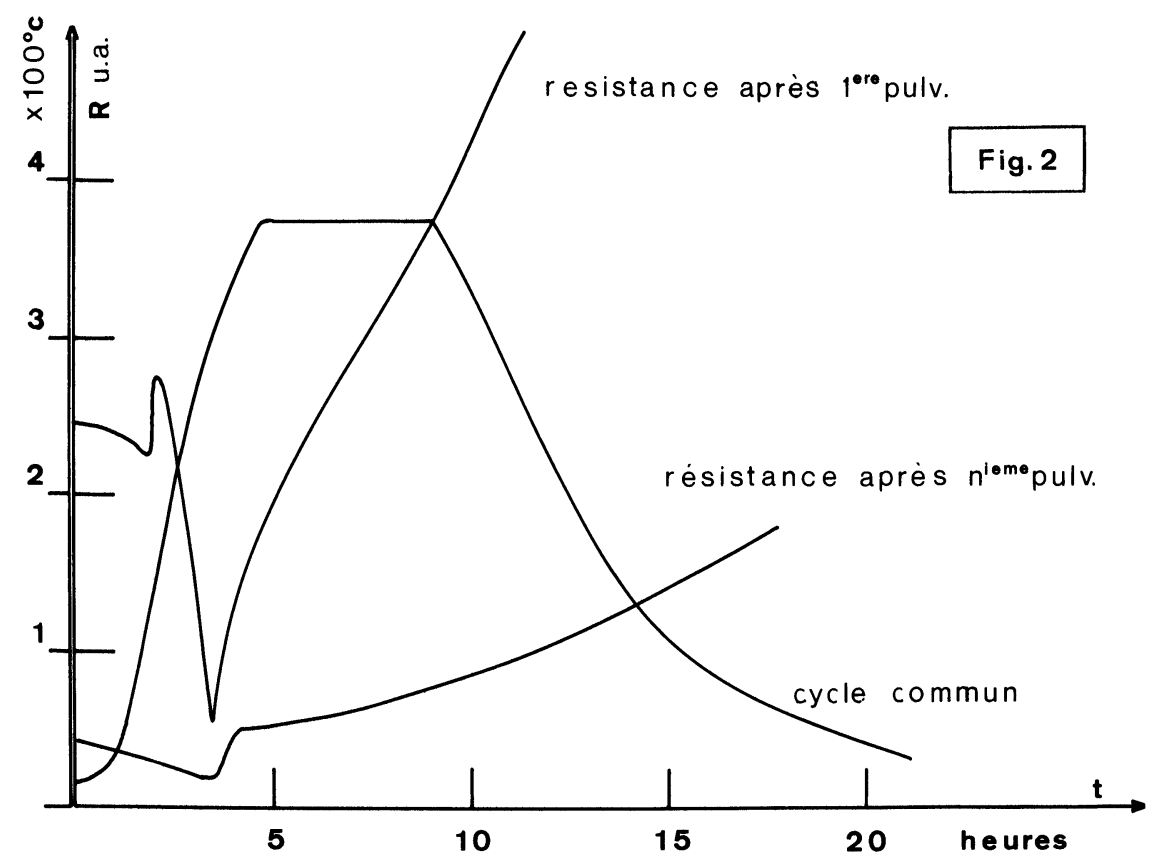

FIGURE 2 Variation, en fonction du temps et de la température, de la résistance (en unités arbitraires) du même substrat après une pulvérisation et après $n$ pulvérisations.

On en déduit un coefficient de température faiblement négatif de l'ordre de $-5 \times 10^{-3} /{ }^{\circ} \mathrm{C}$ au voisinage de la température ambiante, dont l'ordre de grandeur est en bon accord avec les valeurs habituelles concernant des couches préparées dans des conditions différentes ${ }^{35}$.

Cependant, les couches assez minces obtenues après une pulvérisation unique présentent une pente plus importante, correspondant à une énergie d'activation de $0,1 \mathrm{eV}$.

\subsection{Vieillissement spontane a l'air}

En vue d'utilisations éventuelles de ces couches, nous avons été amenés à examiner le vieillissement dans de l'air de degré hygrométrique variant entre $20 \%$ et $40 \%$.

Nous avons d'abord noté que de l'oxygène sec à température élevée $\left(320^{\circ} \mathrm{C}\right)$ et à faible pression $\left(10^{-1}\right.$ Torr $)$ n'a aucune action mesurable sur la résistance des couches, même pendant $30 \mathrm{~min}$.

Mais il suffit de laisser séjourner les couches à l'air libre et à la température ambiante pour observer une décroissance notable de la résistance au cours des premières heures, qui conduit au bout de $72 \mathrm{~h}$ environ à une valeur limite stable. La Figure 4 représente les variations globales de la conductance des couches obtenues par pulvérisations successives en fonction du temps de séjour dans une atmosphère à 30\% d'hygrométrie; le chiffre en paramètre sur les courbes indiquant le nombre de pulvérisation de même durée effectuées pour obtenir la multi-couche, chaque couche intermédiaire ayant subi le vieillissement.

Nous remarquons que le vieillissement à l'air libre produit une décroissance spontanée de la résistance qui est d'autant plus importante que le nombre de pulvérisations successives est plus faible.

La variation de la résistance en fonction du temps n'est pas exponentielle et peut être représentée, dans le domaine d'évolution notable, par une loi temporelle du type

$$
R=R_{0}\left(t / t_{0}\right)^{-\alpha}
$$

où $R_{0}$ se situe entre 17 et $5 \times 10^{3} \Omega$ et $\alpha$ entre 0,4 et 0,5 .

Nous avons observé que le coefficient de température varie de moins de $10 \%$ à la suite de ce vieillissement, ce qui confirme le caractère superficiel des phénomènes correspondants. 


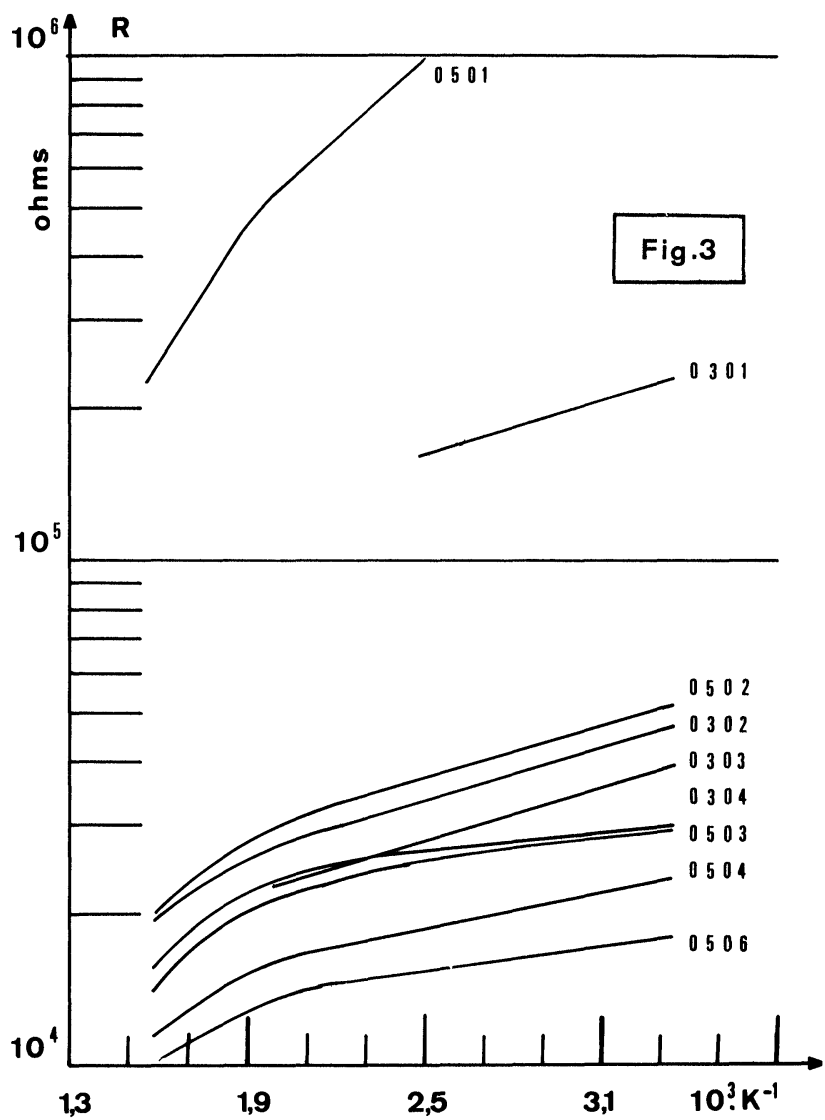

FIGURE 3 Evolution de la résistance $(\log )$ en fonction de la température absolue de plusieurs couches: série 0300: pulvérisation partielle de $20 \mathrm{~min}$; série 0500: pulvérisation partielle de $10 \mathrm{~min}$; dernier chiffre: nombre de pulvérisations.

\subsection{Transparence optique}

A l'aide d'un monochromateur à réseau fournissant un flux lumineux de longueur d'onde comprise entre $2500 \AA$ et $6500 \AA$, avec une largeur de spectre toujours inférieure à $100 \AA$, nous avons déterminé le facteur de transmission optique des couches de $\mathrm{SnO}_{2}$ de différentes épaisseurs, avec une incertitude maximale de $10 \%$.

Les résultats obtenus (Figure 5) sont en bonne concordance qualitative avec les travaux publiés antérieurement ${ }^{36}$ concernant des couches d'épaisseurs voisines; on peut cependant noter la valeur élevée obtenue pour le facteur de transmission (plus de 60\%), notamment pour des longueurs d'onde supérieures à $2500 \AA$ et pour des épaisseurs de matériau conducteur inférieures à $900 \AA$.

\subsection{Les effets de la duree de la pulverisation}

Une longue durée de pulvérisation, par exemple 90 min, ne permet pas d'obtenir des couches de bonne conductivité; la résistance carrée vaut environ $10^{-6} \Omega /$ sq.

En procédant à une succession de pulvérisations de même durée totale, avec un intervalle de temps de l'ordre de 20 min entre deux pulvérisations successives, sans recuit ni remise à l'air intermédiaires, la résistance carrée est plus faible; elle atteint $10^{4} \Omega /$ sq.

Des résistances de l'ordre de $10^{3} \Omega / \mathrm{sq}$. sont obtenues si on procède à un recuit et à un vieillissement à l'air après la pulvérisation de chaque couche constituant la multi-couche.

Sur la Figure 6 nous avons tracé les variations successives de la conductance de quelques multicouches, quant on procède à un recuit et à un vieillissement à l'air libre après chaque pulvérisation; les 3 courbes tracées correspondent à des pulvérisations élémentaires de durées égales respectivement à 20,30 et $10 \mathrm{~min}$. Lorsque la durée de la pulvérisation élémentaire est supérieure à $15 \mathrm{~min}$, il apparait que la conductance d'une multicouche est généralement accrue par le dépôt d'une couche nouvelle. L'écart de ces courbes par rapport à une loi linéaire indique la non-homogénéité de l'ensemble de la multi-couche. Nous avons également observé qu'il est possible d'obtenir les conductances les plus élevées lorsque la durée de la pulvérisation élémentaire est la plus faible (10 min dans nos expériences).

A notre connaissance, les effets dimensionnels de cette nature n'ont pas été mentionnés par les précédents expérimentateurs qui ont généralement opéré sur des couches d'épaisseur très supérieure à $2000 \AA$.

\section{ESSAI D'INTERPRETATION}

En admettant que le problème de la germination et de la croissance d'une couche mince déposée par pulvérisation peut être ramené à la compréhension des mécanismes de stabilisation d'une entité instable ou métastable synthétisée dans le plasma, Laville Saint Martin 38-40 a montré que la croissance d'une couche mince sur un substrat isolant ne dépend pas de la nature chimique de l'entité jusqu'à l'obtention d'une épaisseur critique qualifiée d'épaisseur critique de première espèce, qui correspond à l'obtention d'une couche continue, et dont la valeur est généralement inférieure à $300 \AA^{40}$; une réaction de 


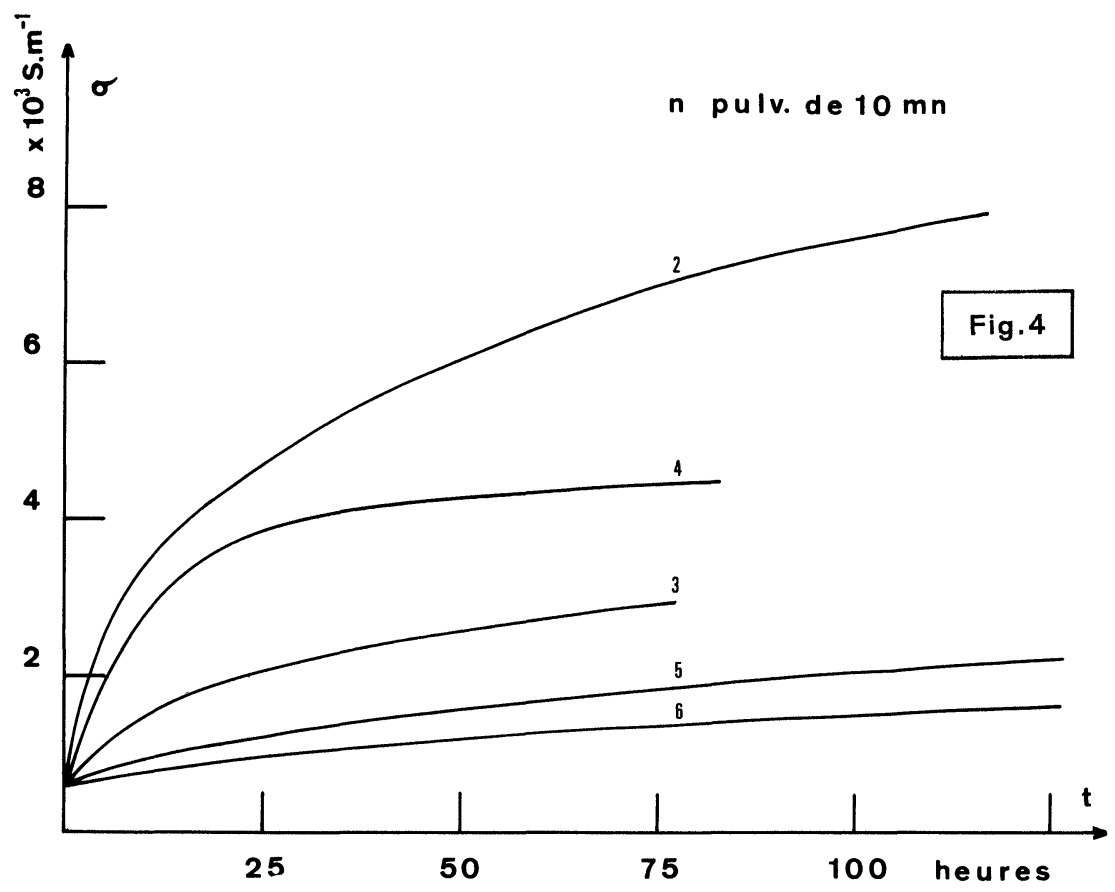

FIGURE 4 Evolution de la conductance d'une couche en fonction du temps d'action de l'air ambiant.

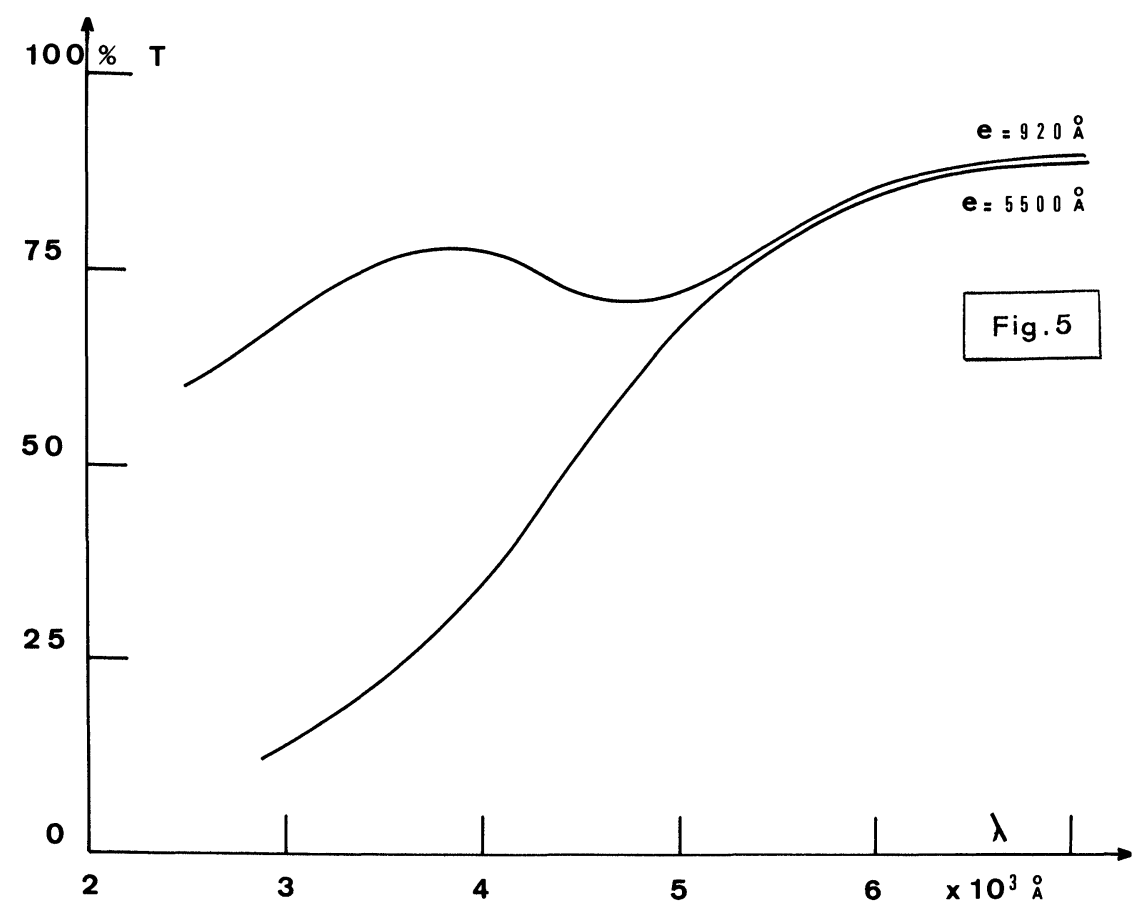

FIGURE 5 Variation du coefficient de transmission en fonction de la longueur d'onde pour deux épaisseurs différentes. 
désexcitation Auger permet la croissance ultérieure ${ }^{40}$, dans la mesure où des électrons sont disponsibles sur le support de condensation, ce qui nous semble compatible avec la nature électrique de la couche conductrice déposée; la couche mince devient ainsi continue et uniforme pour une épaisseur qui est qualifiée d'épaisseur critique de deuxième espèce, et dont la valeur se situe entre $600 \AA$ et $900 \AA^{39}$ dans le cas de couches métalliques pulvérisées sous tension continue. Au-delà de cette épaisseur, il se produit une délocalisation des sites de germination qui conduit à une phase de croissance qualifiée "d'iso-épitaxique" 40 au cours de laquelle la quantité de matière condensée est fonction linéaire du temps et la croissance s'effectue avec un degré d'ordre élevé 40 ; en conformité avec ces hypothèses, nous obtenons effectivement un taux de pulvérisation constant, dès que l'épaisseur de la couche conductrice dépasse $300 \AA$; cette valeur est très inférieure aux valeurs citées; ceci nous paraît indiquer que la bonne définition géométrique du plasma, autorisée par la tension haute fréquence, délimite nettement la zone privilégiée de source de matière condensable qui fut qualifiée de "couche virtuelle" 39 et permet une uniformisation plus rapide de la surface de la couche condensée. D'ailleurs nous avons déjà remarqué, dans le cas de couches minces isolantes de sulfure de zinc déposées sur une languette d'aluminium ${ }^{33}$, que la pulvérisation cathodique haute-fréquence conduisait à une structure microcristalline correspondant à des grains de structure cubique, de dimension voisine de $50 \AA^{37}$ et que, sauf au cours des premières minutes de pulvérisation, le taux de pulvérisation était constant.

Nous sommes donc amenés à penser que la croissance quasi-isoépitaxique de ces couches, qui est initialement permise par la désexcitation Auger au contact des électrons du métal, se poursuit ensuite d'une facon régulière car la répartition des sites de germination est détérminée par la couche sousjacente; l'homogénéité de ces couches étant observée dans un domaine d'épaisseur s'étendant de $190 \AA$ à $650 \AA$, nous supposons que ce procédé de pulvérisation dégrade très lentement l'ordre, d'une couche, qu'elle soit conductrice ou isolante.

Cependant comme la couche conductrice déposée n'est pas métallique, la désexcitation Auger peut ne pas toujours d'opérer dans des conditions permettant une stricte conservation de l'ordre iso-épitaxique et nous supposons qu'à mesure que l'épaisseur déposée croît, cet ordre se dégrade peu à peu, comme le montre la décroissance de la conductivité avec la durée de la première pulvérisation élémentaire (Figure 6).
Le recuit sous vide, qui permet notamment une restructuration mécanique de la couche ${ }^{32}$ et l'évolution spontanée à l'air libre, qui permet des réactions superficielles d'oxydation, ont pour effet d'améliorer le degré d'ordre de la couche, ce qui conduit à une diminution de la résistance; le réajustement du degré d'ordre peut être d'autant plus important que sa dégradation était plus grande; nous observons effectivement une plus grande diminution de la résistance lors du recuit si la durée de la pulvérisation est plus longue.

De même le dépôt de couches superposées, soumises après chaque pulvérisation élémentaire à un recuit et à une évolution spontanée, conduit à une couche mieux ordonée que la couche de même épaisseur totale déposée en une seule opération de pulvérisation et une amélioration de la conductivité est obtenue à chaque nouveau dépôt. Cependant si la première couche est assez mince pour avoir conservé un degré d'ordre notable, sa conductivité est peu affectée par le recuit et le dépôt d'une deuxième couche dégrade cet ordre dans des conditions telles qu'il ne peut être rétabli après recuit, on observe donc une diminution apparente de conductivité lors du dépôt de couches nouvelles sur une mince couche initiale, ce qui est bien conforme aux observations expérimentales et notamment à l'existence d'un maximum de conductivité (Figure 6, courbe supérieure). La résistance de cette couche mince demande d'ailleurs une plus forte activation pour évoluer thermiquement que les couches de plus forte épaisseur, ce qui est bien cohérent avec l'hypothèse concernant les degrés d'ordre.

Dans le cas des expérimentations présentées, un degré d'ordre élevé est obtenu lorsque la durée de la pulvérisation élémentaire ne dépasse pas $20 \mathrm{~min}$ ce qui correspond à une épaisseur voisine de $1800 \AA$ et à une résistance carrée de $27 \mathrm{k} \Omega$.

Les considérations précédentes nous conduisent donc à associer à un degré d'ordre élevé une forte conductivité et, à un degré d'ordre faible, une faible conductivité. Les conductivités comparées des couches superposées ou non et les phénomènes de recuit mettent en évidence l'importance de la surface supérieure; nous sommes ainsi amenés a postuler l'existence de phénomènes du type Fuchs-Sondheimer et à associer au degré d'ordre, de valeur forte ou faible, une valeur forte ou faible du coefficient de réflexion spéculaire sur l'interface supérieure, rejoignant ainsi les hypothèses avancées par plusieurs auteurs dans des cas similaires ${ }^{4-46}$; une décroissance du paramètre $p$ de réflexion spéculaire sur l'interface supérieure avec l'épaisseur totale de la couche peut ainsi rendre 


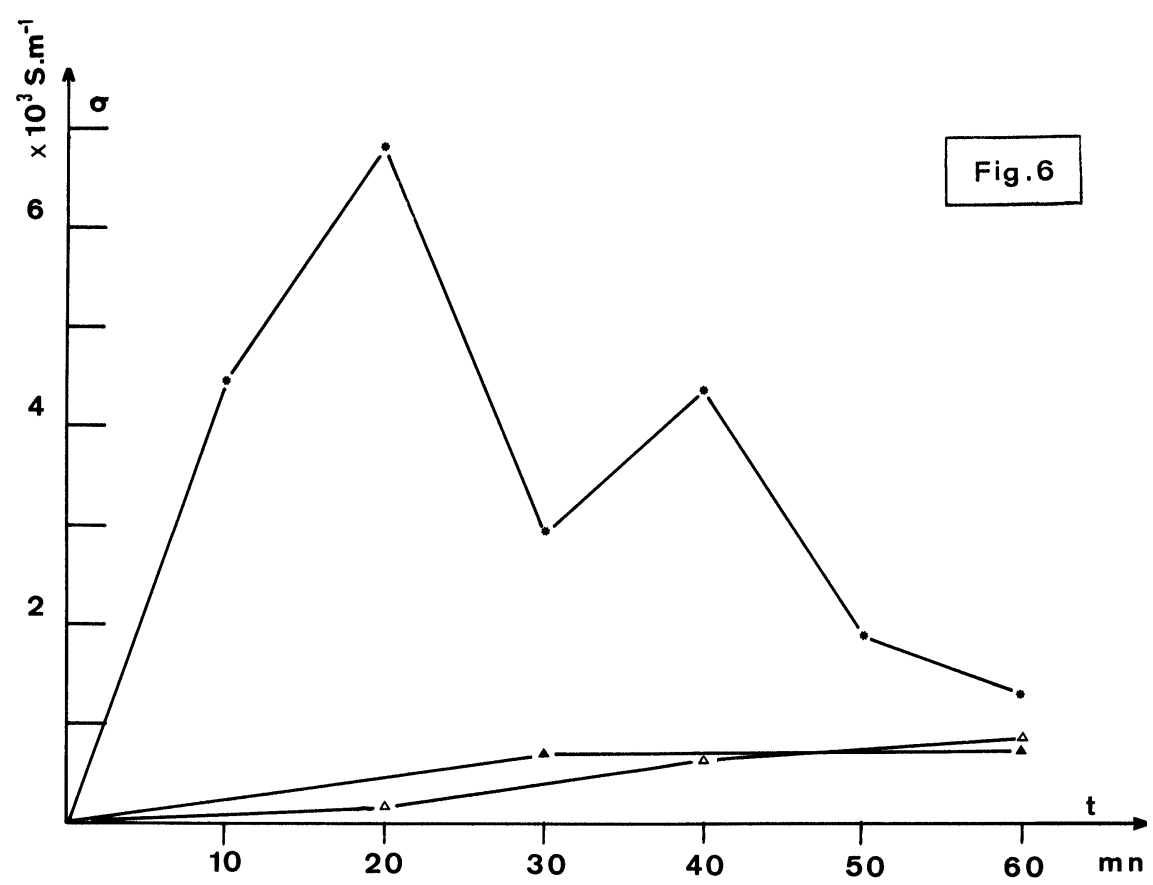

FIGURE 6 Valeurs successives de la conductance de multicouches pour trois périodicités de pulvérisations élémentaires. $\triangle 30 \mathrm{~min} ; \triangle 20 \mathrm{~min}$; * $10 \mathrm{~min}$.

compte de la diminution de conductivité observée par dépôt d'une couche nouvelle sur une couche assez mince pour présenter un degré d'ordre élevé, donc un facteur de réflexion spéculaire notable 42 .

Les singularités dimensionnelles observées pour les couches pulvérisées en haute fréquence sont donc bien cohérentes avec l'hypothèse de germination croissance des couches métalliques ${ }^{40}$. Une amélioration technologique des procédés de fabrication de couches minces conductrices et transparentes nous paraît ainsi pouvoir être recherchée de facon systématique.

\section{CONCLUSION}

L'étude dimensionnelle des propriétés de conductivité et de transparence optique de couches obtenues par pulvérisation réactive haute fréquence d'une cible de $\mathrm{SnO}_{2}$ permet de définir les conditions de pulvérisation conduisant à une transparence optique supérieure à $60 \%$ au-dessus de $2500 \AA$, tout est maintenant la résistance carrée à un niveau acceptable inférieur à $1 \mathrm{k} \Omega / \mathrm{sq}$.

\section{REFERENCES}

1. K. Tanaka, Jap. J. Appl. Phys., 8, 6, 681 (1969).

2. R. R. Mehta and S. F. Vogel, J. Electrochem. Soc., 119, 9, 752 (1972).

3. G. Kienel and G. Gallus, Jap. J. Appl. Phys., Suppl. 2, Pt. 1, p. 479 (1974).

4. M. Hecq, A. Dubois and J. Van Cakenberghe, ler Colloq Int. Pulv. Cath., Montpellier, p. 151 (1973).

5. E. Leja, Acta Physica Polonica A., 38A, 2, 165 (1970).

6. J. L. Vossen, R.C.A. Reviero, 52, 2, 289 (1971).

7. D. B. Fraser and H. D. Cook, Extended Abstracts of the Spring Meeting of the Electrochem. Soc., Washington D.C. 3-13 May, p. 76 (1971).

8. J. L. Vossen and E. S. Poliniak, Thin Solid Films, 13, 281 (1972).

9. D. B. Fraser and H. D. Cook, J. Electrochem. Soc., 119, 10, 1368 (1972).

10. M. M. Bonnet-Marchal, ler Colloq. Int. Pulv. Cath., Montpellier, p. 157 (1973).

11. M. Hecq, A. Dubois and J. Van Cakenberghe, Thin Solid Films, 18, 1, 117 (1973).

12. J. R. Rosnel, Thin Solid Films, 15, 2, 141 (1973).

13. Libbey-Owens-Ford Glass Co and H. A. McMasters, Brit. Pat. 632,256.

14. Libbey-Owens-Ford Glass Co, Brit. Pat. 682,342.

15. R. Gomer, Rev. Sci. Instrum., 24, 993 (1953).

16. J. A. Aboaf, V. C. Marcotte and N. J. Chou, J. Electrochem. Soc., 120, 5, 701 (1973). 
17. F. V. D. Maesen and C. H. M. Witmer, Proc. 7th Intern. Conf. on the Phys. of Semi-conductors Paris, p. 1211 (1964).

18. R. Colin, J. Droward and G. Verhaegen, Trans Faraday Soc., 61, 1364 (1965).

19. M. Shiogiri, H. Motikawa and E. Suite, Sixth Intern. Cong. for Electron Microscopy, p. 467.

20. Anonymous, Nature, 169, 829 (1952).

21. L. Holland and G. Siddall, Vacuum, III, 4 (1953).

22. W. R. Sinclair and F. G. Peters, J. Am. Ceram. Soc., 46, 1 (1963).

23. W. R. Sinclair, F. G. Peters, D. W. Stillinger and C. E. Koon, J. Electrochemical Soc., 112, 11, 1096 (1965).

24. V. M. Vainshtein, Simp. Protsessy Sin Rosta Krist Plenok Prolupov Mater Tezisy Dokl, p. 296 Novasibirsk (1965).

25. J. Baillou, P. Bugnet, J. Deforges, S. Durand and G. Batailler, Rev. Phys. Appl., 3, 78 (March 1968).

26. M. Liebermann and R. C. Medrud, J. Electrochem. Soc., 116, 242 (1969).

27. E. Leja, Acta Phys. Pol. A., 38, 2, 165 (1970).

28. M. Hecq and E. Portier, Thin Solid Films, 9, 341 (1972).

29. J. L. Vossen and E. S. Poliniak, Thin Solid Films, 13, 281 (1972).

30. Yamanaka and Oohashi, Jap. J. Appl. Phys., 8, 1058 (1969).

31. L. Holland, Vacuum Deposition of Thin Films,
Chapter 14, John Wiley and Sons Inc., New York (1956).

32. A. Tosser, G. Fleury and H. Murray, Thin Solid Films, 15, 259 (1973).

33. H. Murray and A. Tosser, Thin Solid Films, 17, 75 (1973).

34. E. Giani and R. Kelly, J. Electrochem. Soc., 121, 3 (1974).

35. K. L. Chopra, Thin Film Phenomena, Chapter VI, McGraw-Hill, New York (1969).

36. J. P. Cebe and J. M. Mathieu, C.R. Acad. Sc. Paris, t. 270, $111(1970)$

37. H. Murray and A. Tosser, Thin Solid Films, 24, 165 (1974).

38. B. Laville St Martin, Thin Solid Films, 4, 319 (1969),

39. B. Laville St Martin, Thin Solid Films, 5, 169 (1970).

40. B. Laville St Martin, Thin Solid Films, 5, 359 (1970).

41. T. J. Coutts, Thin Solid Films, 7, 77 (1971).

42. M. S. P. Lucas, Thin Solid Films, 2, 337 (1968).

43. M. S. P. Lucas, Appl. Phys. Lett., 4, 73 (1964).

44. J-P. Chauvineau and C. Pariset, Surf. Sci., 36, 155 (1973).

45. A. K. Pal, S. Paraminta and A. K. Barva, Thin Solid Films, 25, S25 (1975).

46. T. T. Sheng, R. B. Marcus, F. Alexander and W. A. Reed, Thin Solid Films, 14, 289 (1972). 

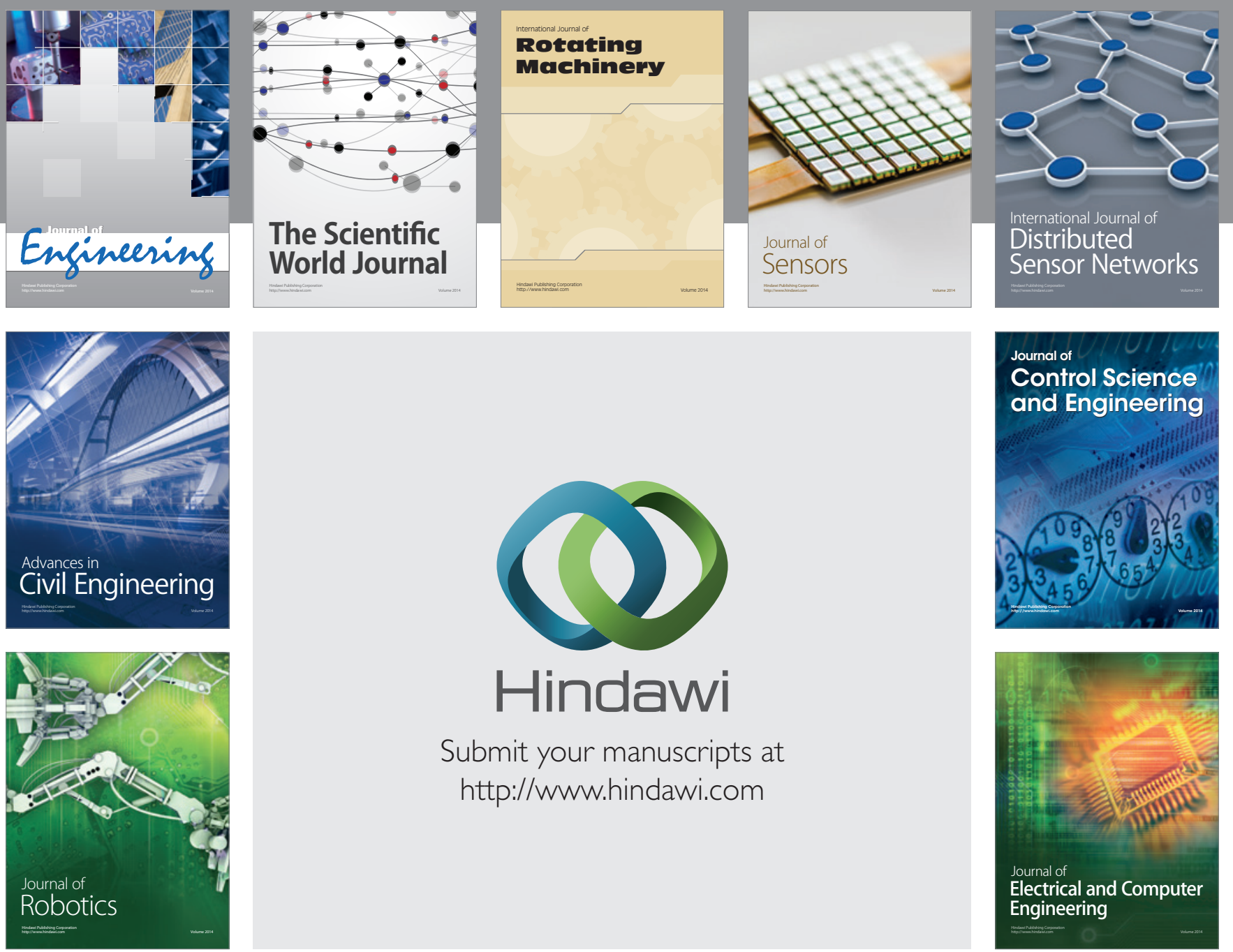

Submit your manuscripts at

http://www.hindawi.com
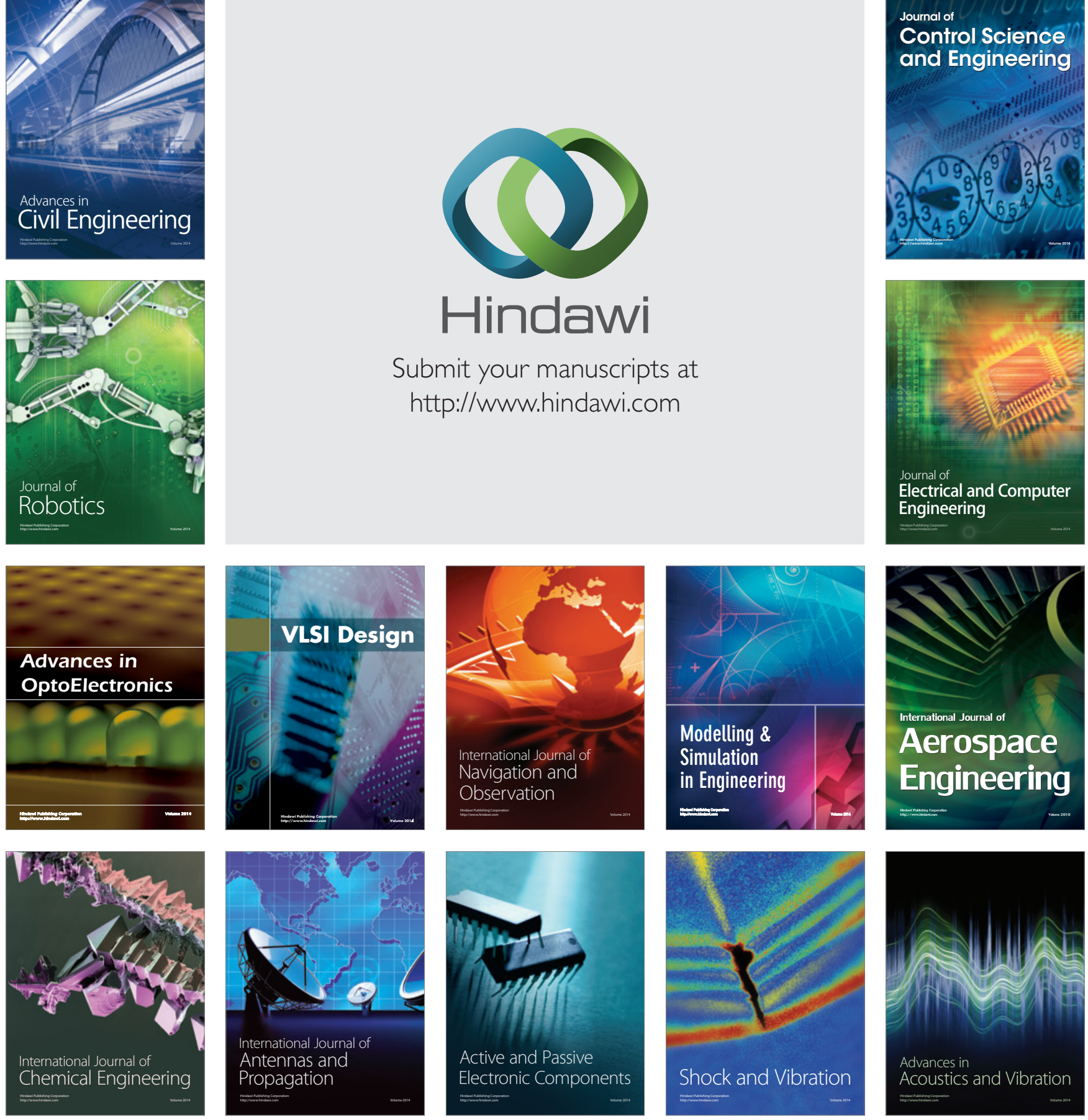